\title{
Environmental magneto-gradiometric marine survey in a highly anthropic noisy area
}

\author{
Davide Embriaco $\left({ }^{1}\right)$, Cosmo Carmisciano $\left({ }^{1}\right)$, Fabio Caratori Tontini $\left({ }^{2}\right)$, Paolo Stefanelli $\left({ }^{1}\right)$, \\ Luca Cocchi ( $\left.{ }^{1}\right)$, Marina Locritani $\left({ }^{1}\right)$ and Maurizio De Marte $\left(^{3}\right)$ \\ (') Istituto Nazionale di Geofisica e Vulcanologia, Portovenere (SP), Italy \\ ${ }^{2}{ }^{2}$ Istituto Nazionale di Geofisica e Vulcanologia, Portovenere (SP), \\ Italy now at GNS Science, Lower Hutt, New Zealand \\ $\left({ }^{3}\right)$ Istituto Idrografico della Marina Militare, Genova, Italy
}

\begin{abstract}
We describe a magneto-gradiometric survey performed in the «Mar Piccolo» of Taranto, Italy in May 2005 for environmental purposes. This region, which is a noisy harbour environment, provides a challenging test for magnetic methods. To reduce spurious noise signals, with both temporal and spatial origins, we used two Geometrics G880 model caesium magnetometers towed in a transverse gradient configuration. We show how, in shallow waters, this gradiometric configuration allows us to distinguish anomalies due to small metallic bodies near the seabed from the induced noise due to the anthropic contribution and geomagnetic field variations. A direct visual inspection confirmed that the peculiarities highlighted in the gradient anomaly map were due to abandoned metallic objects found on the seabed.
\end{abstract}

Key words environmental reclamation - anthropic noise - magneto-gradiometric survey

\section{Introduction}

Coastal habitats are characterised by a delicate equilibrium due to the interactions of multiple factors; in harbour zones in particular, they are strongly modified and affected by anthropic impacts. The development of infrastructure and harbour traffic cause frequent destabilisation to these habitats, so human interventions to safeguard them are becoming increasingly common. Reclamation and reorganisation are the starting points to regain adequate environmen-

Mailing address: Dr. Davide Embriaco, Istituto Nazionale di Geofisica e Vulcanologia, Via Pezzino Basso 2, 19025 Portovenere (SP), Italy; e-mail: davide.embriaco@ingv.it tal sustainability and can be achieved by eliminating pollution sources and polluting substances or at least reducing the actual concentrations in the various environmental scenarios. The first phase of a reclamation process and environmental restoration is to classify, quantify and locate abandoned or uncontrolled waste.

A large number of metallic objects can be found in a harbour area: barrels, which may have been lost during naval operations or intentionally abandoned, chains, and even weapons from the last war. Thus, the first step in a thorough investigation is a magnetic survey of the area, which can identify the magnetic signals of these objects and help to locate their positions. Magnetic surveys are largely used to produce a high-resolution characterisation of shallow water (Mansoor et al., 2006). They are the preferred prospecting method for locating submerged metallic objects (Ravat, 1996) and can be helpful in searching for underwater cultural 
resources (Caratori Tontini et al., 2006; Boyce and Reinhardt, 2001), and can also be useful for mapping the contaminated sediment distribution in harbour areas (Pozza et al., 2004). A magnetic survey can reveal not only metallic objects on the seabed, but also targets below it: this turns out to be very important if the targets are buried in the mud and thus hidden to a visual inspection. Some surveys in shallow water have shown the suitability of magnetic methods for searching for lost artefacts (Weiss et al., 2007) or for mapping and locating large amounts of small abandoned metallic objects (Hrvoic and Pozza, 2006).

Within this context, we performed a magnetic survey during May 2005 in the «Mare Piccolo» of Taranto, Italy. The «Mar Piccolo» is a half-closed river basin of approximately 20 square $\mathrm{km}$ that is divided by two capes into two smaller basins, one of which is connected to the «Mar Grande», and hence to the Ionian Sea, through two channels; the second is more inland and has only a slight marine circulation. The «Mare Piccolo» is surrounded by the city of Taranto, which is a significant environmental hazard industrial area and, being seriously compromised in all environmental sectors, is considered a «site of national interest for reclamation actions» (Parlamento italiano, 1998; Ministero ambiente e tutela del territorio, 2001). The selected area is characterised by high magnetic noise, while the survey aimed to find small metallic objects on the seabed at a depth of up to 15-20 m; thus, the main task of this survey was to distinguish small magnetic anomalies among the anthropic magnetic induced noise typically present in a commercial harbour area. We thus set up a gradiometric magnetic survey in the area under investigation. The hope was that the relative difference between the two sensors would dramatically reduce the effect of spurious signals coming from temporal variations or other anomalies of anthropic origin that are largely suppressed at the scale of a few meters.

We used two magnetometers in a transverse gradient configuration, allowing us to directly measure the horizontal components of the total field magnetic gradient. In a limited area of the survey zone, we measured both horizontal components of the gradient of the total magnetic field, creating an orthogonal grid of survey lines. This enabled us to directly compute and inspect the Analytic Signal (AS) (Nabighian 1972 ; 1974) of the magnetic data, taking advantage of its mathematical properties (Nabighian, 1984; Roest et al., 1992; Pedersen, 1989; Nelson, 1986) to obtain information on the anomalies, including an estimation of the depth of the source. The AS was computed starting directly from the data measured by the gradiometric device rather than being derived from a numerical evaluation of the total field derivatives using magnetic anomaly data. This procedure assures that artefacts due to the noisy area are automatically suppressed and that the signal to noise ratio increases. Moreover, the gradiometric data can give direct information during the field operation without the need for long post-processing; this can enhance the speed of information gathering for the target identification, a feature that can be important for quick reclamation processes.

In the following sections, we describe the planning procedure for the survey and the magneto-gradiometric configuration, and then show the results obtained and discuss the advantages of direct gradiometic data processing as regards the analysis of the magnetic anomaly in a noisy area.

\section{Data acquisition}

The survey area was covered via a mesh made as regular as possible, comprising a set of parallel lines and orthogonal tie lines. The survey geometry, in terms of line spacing, and the sampling rate were chosen to facilitate detection of the expected anomalies, while also taking into account the available shipping schedule. The Italian Navy «Istituto Idrografico della Marina» (IIM) supplied all of the marine logistics needed to perform the present survey, specifically, vessels and magnetometers. Thus, accurate sensor positioning was guaranteed by the use of the differential global positioning system (DGPS) that is currently installed onboard IIM vessels. A reference magnetic base station was placed near the survey area in order to monitor the Earth's geomagnetic field. A 
good knowledge of the depth in the survey area was obtained using an IIM multibeam survey. The coastline and a profile of harbour structures were also delineated (see fig. 1).

Extreme conditions for the magnetic survey were found in the area: these were due to the remarkable presence of anthropic noise caused by the large number of ships and harbour infrastructures. The particular noisy condition necessitated the planning of a magneto-gradiometric survey that can highlight local magnetic sources while suppressing the contribution of regional variations in the anomaly data. The gradiometer system comprised two caesium magnetometers (Geometrics G 880 model) that acquired two total magnetic field values at two points separated by two meters. The difference between the two magnetic values divided by the distance between the sensors is a good approximation for the component of the total magnetic field gradient along the direction parallel to the sensor separation.

The gradiometric device was towed in a rubber dinghy by a glass reinforced plastic hydrographic boat at a distance of $20 \mathrm{~m}$. Such a distance represents a good compromise among the requirements of minimising the effects of noise generated by the ship and its equipment, the need to limit the positioning errors due the positional inconsistencies associated with ropetowing and the need to keep the headings of the two sensors as constant as possible. The placement of the two sensors immediately under the hull of the rubber dinghy was due to the difficulties associated with manoeuvring the boat and the towing.

The survey was planned with east-west profiles with an inter line distance of approximately $20 \mathrm{~m}$. Furthermore, twelve ties were acquired with variable inter-line separation to refine the identification of magnetic signatures found during the line acquisition in the area highlighted by the square white box in fig. 1 . The whole survey area included approximately 1.7 square $\mathrm{km}$ and was covered by approximately $65 \mathrm{~km}$ of data acquisition tracks. During the survey, all of the observed surface objects in the measured area (buoys, catenaries, ships, etc.) were taken into account so their signals could be identified during the processing step.

\section{Results}

The direct availability of the gradient signal makes it possible to produce a map of gradient magnetic anomalies in an area quickly without the need to heavily process the data. The gradient signal is not affected by the temporal variations of the geomagnetic field, and the noise due to anthropic activities is strongly suppressed: thus, this technique can provide quick and direct information for the locating the sources of signal anomalies in the area. Two kinds of magnetic data were directly available for the present survey geometry: the northsouth component of the total magnetic field gradient in the area covered by the east-west lines, hereafter called $M_{y}$, and the east-west component in the area also covered by the north-south tie lines, hereafter called $M_{x}$. The gradiometric signal maps are plotted in fig. 2 for $M_{y}$ and fig. 3 for $M_{x}$. Due to a lack of ship time, we were not able to cover the whole area in both directions (north-south and east-west), so the $M_{x}$ component was evaluated only in a small zone of the whole area. The boxed area on the left in fig. 2 shows the overlap between the two surveys (lines and ties). In this boxed area, it is also possible to compute the norm of the 3D analytical signal of the magnetic field, defined as

$$
|A(x, y)|=\sqrt{\left(\frac{\partial M}{\partial x}\right)^{2}+\left(\frac{\partial M}{\partial y}\right)^{2}+\left(\frac{\partial M}{\partial z}\right)^{2}}
$$

where $M$ is the total field anomaly, the positive direction $x$ is toward the east, the positive direction $y$ is toward the north and $z$ is along the vertical. The first two terms of the previous quantities are directly measured, while the last can be computed from $M_{x}$ and $M_{y}$ using the known relation (Nabighian, 1984)

$$
F\left[M_{z}\right]=-\frac{i k_{x}}{k} F\left[M_{x}\right]-\frac{i k_{y}}{k} F\left[M_{y}\right]
$$

where $F[\ldots]$ is the Fourier transform operator, $k_{x}$ and $k_{y}$ are the wave vectors in Fourier space and $k \equiv \sqrt{k_{x}^{2}+k_{y}^{2}}$. The map of the 3D analytical signal is shown in fig. 4 .

The analysis of the gradient map shows clear anomalies related to the coast line; these are 


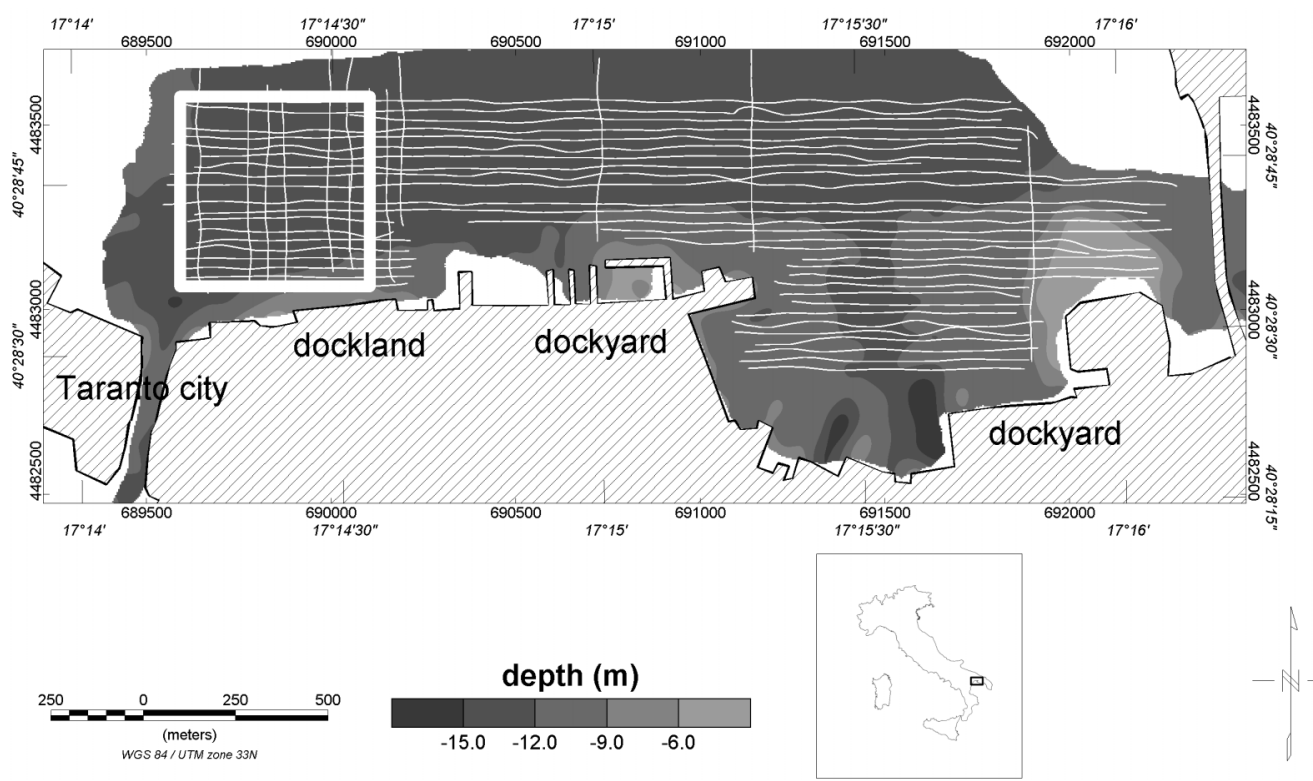

Fig. 1. Bathymetry of the survey area, main harbour infrastructure. The Taranto harbour is located on the southeastern coast of Italy, approximately in the middle of the box on the bottom right map. The survey extent lines and ties path are indicated in white; in the boxed region on the left, all the horizontal components of the magnetic gradient are measured and the AS is evaluated.

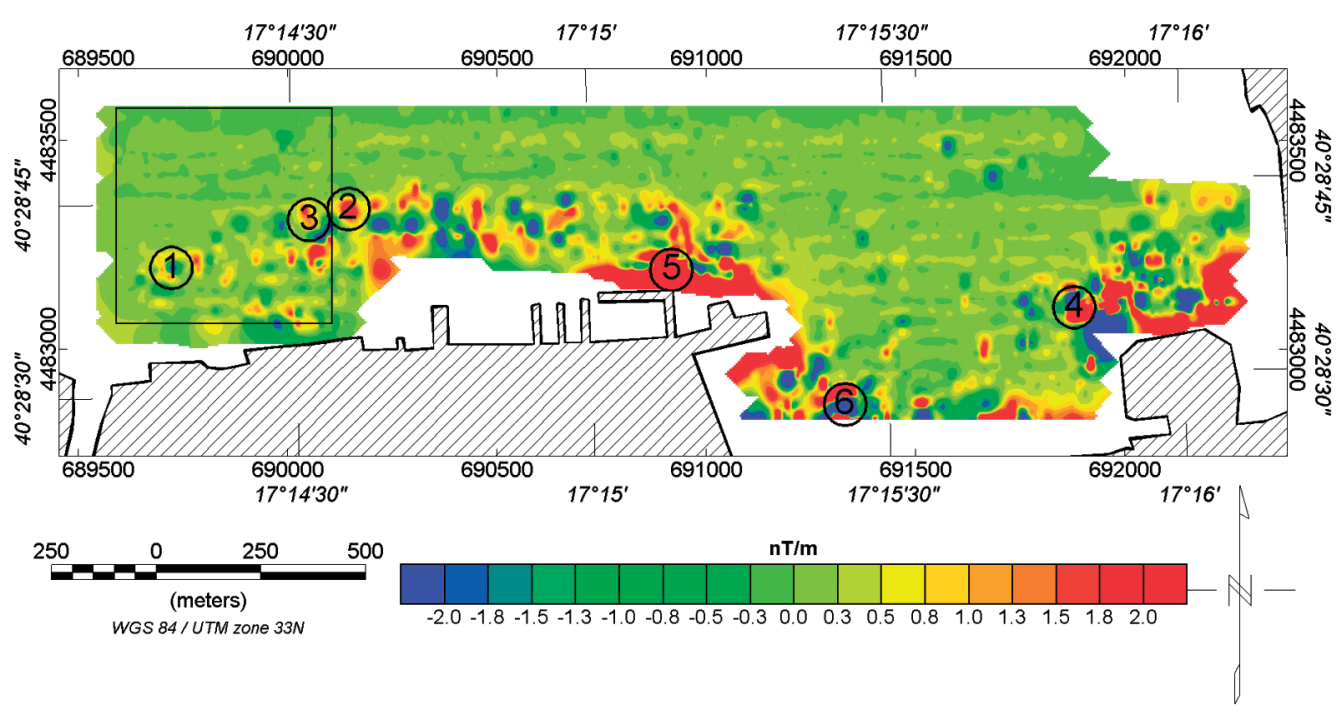

Fig. 2. Magnetic gradient: measured component in the north-south direction; the circles identify the actual object found on the seabed and listed in table I. The whole analytical magnetic signal was computed inside the box on the left. This is plotted in fig. 4. 


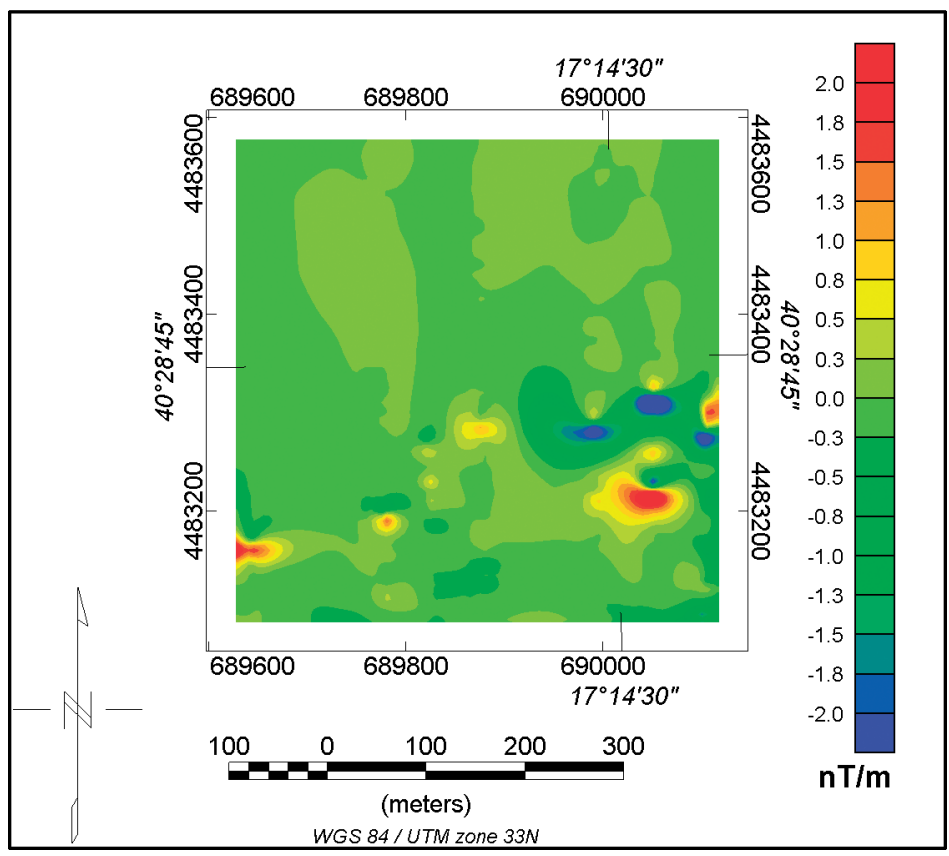

Fig. 3. Magnetic gradient: the east-west component.

Table I. Relevant points and metallic objects identified via visual inspection.

\begin{tabular}{cccc}
\hline \hline ID & LONG & LAT & Visual identification \\
\hline 1 & $17^{\circ} 14^{\prime} 17.3^{\prime \prime}$ & $40^{\circ} 28^{\prime} 39.4^{\prime \prime}$ & Catenary \\
2 & $17^{\circ} 14^{\prime} 35.8^{\prime \prime}$ & $40^{\circ} 28^{\prime} 43.8^{\prime \prime}$ & Iron-filled tire \\
3 & $17^{\circ} 14^{\prime} 31.3^{\prime \prime}$ & $40^{\circ} 28^{\prime} 44.1^{\prime \prime}$ & One-armed anchor \\
4 & $17^{\circ} 15^{\prime} 48.9^{\prime \prime}$ & $40^{\circ} 28^{\prime} 35.2^{\prime \prime}$ & Small metallic pieces \\
5 & $17^{\circ} 15^{\prime} 08.2^{\prime \prime}$ & $40^{\circ} 28^{\prime} 38.9^{\prime \prime}$ & Iron \\
6 & $17^{\circ} 15^{\prime} 25.5^{\prime \prime}$ & $40^{\circ} 28^{\prime} 28.1^{\prime \prime}$ & Iron \\
\hline
\end{tabular}

mostly due to the signal induced by the ships and harbour structures, with the exception of point 6 , which reveals a high intensity not compatible with the superficial evidence. Point 5 turns out to be near a wharf that is under construction, and the northwards elongated anomaly is probably due to a source that goes towards the north and may be linked to such superficial objects. In the north-east area, there are small anomalies due to the presence of surface buoys with an attached chain identified during the survey. The other anomalies do not appear linked to any superficial objects, and were therefore selected for a further investigation using direct visual techniques. Scuba divers examined a subset of the selected locations and identified the signal sources at the measured points; these are summarised in table I. 


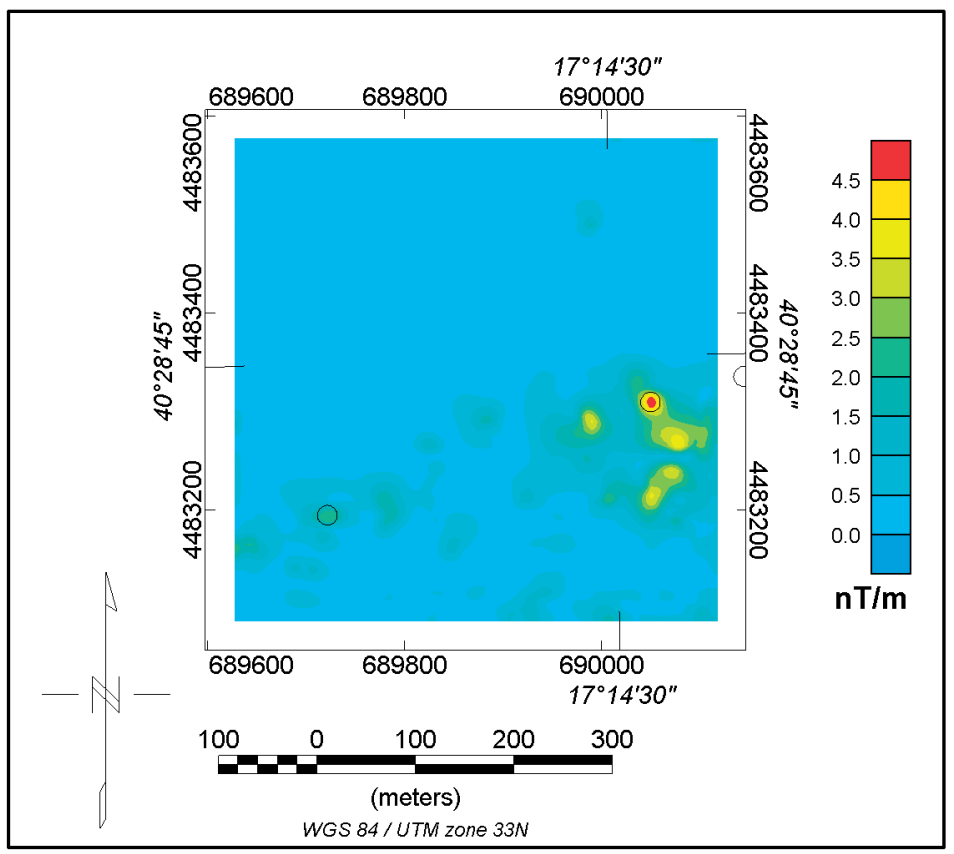

Fig. 4. 3D magnetic analytic signal norm calculated in the boxed area of fig. 2 using gradiometric data.

\section{Discussion}

The advantage of processing gradient data instead of the magnetic signal is clear if we look at a single line profile as shown in fig. 5 . This plot highlights how the magnetic profile shows anomalies due not to magnetic sources like the searched targets on the seabed (see for instance the anomalies on the right part of the panel), but probably to natural or anthropic induced variations: the gradient signal is not affected by these noisy contributions. To further highlight the difference between the two approaches to data analysis in the Taranto area with its substantial anthropic noise, the left panel of fig. 6 presents the time reduced magnetic anomaly evaluated in the square box drawn in figs. 1 and 2, as measured by the two sensors. It is clear that the magnetic anomaly contains very noisy components; it can be difficult to distinguish between the desired signal and the noise or even survey artefacts on that map. Thus, target localisation can be problematic.
The right panel of fig. 6 shows the Analytical Signal, computed using the magnetic anomalies, as these were the only available data: here, the $M_{x}$ and $M_{y}$ contributions to AS are evaluated by computing the numerical derivative of the magnetic anomaly data. This could be the result of a survey performed without using a gradiometric configuration in such a noisy area. The difference between this data and that in fig. 4 again demonstrates the difficulty in determining which anomalies are related to real magnetic sources and which are due to artefacts. It is thus clear that it is advantageous to perform a gradiometric survey by directly measuring the gradient or at least some component of it rather than post-processing a magnetic signal to numerically compute the gradient of the field. In the present configuration, the only component that cannot be measured is the vertical one $\left(M_{z}\right)$, which is computed starting with the knowledge of the two horizontal gradient components in the box in fig. 2, using eq. 3.2 to construct the analytical signal as defined in eq. 3.1. 

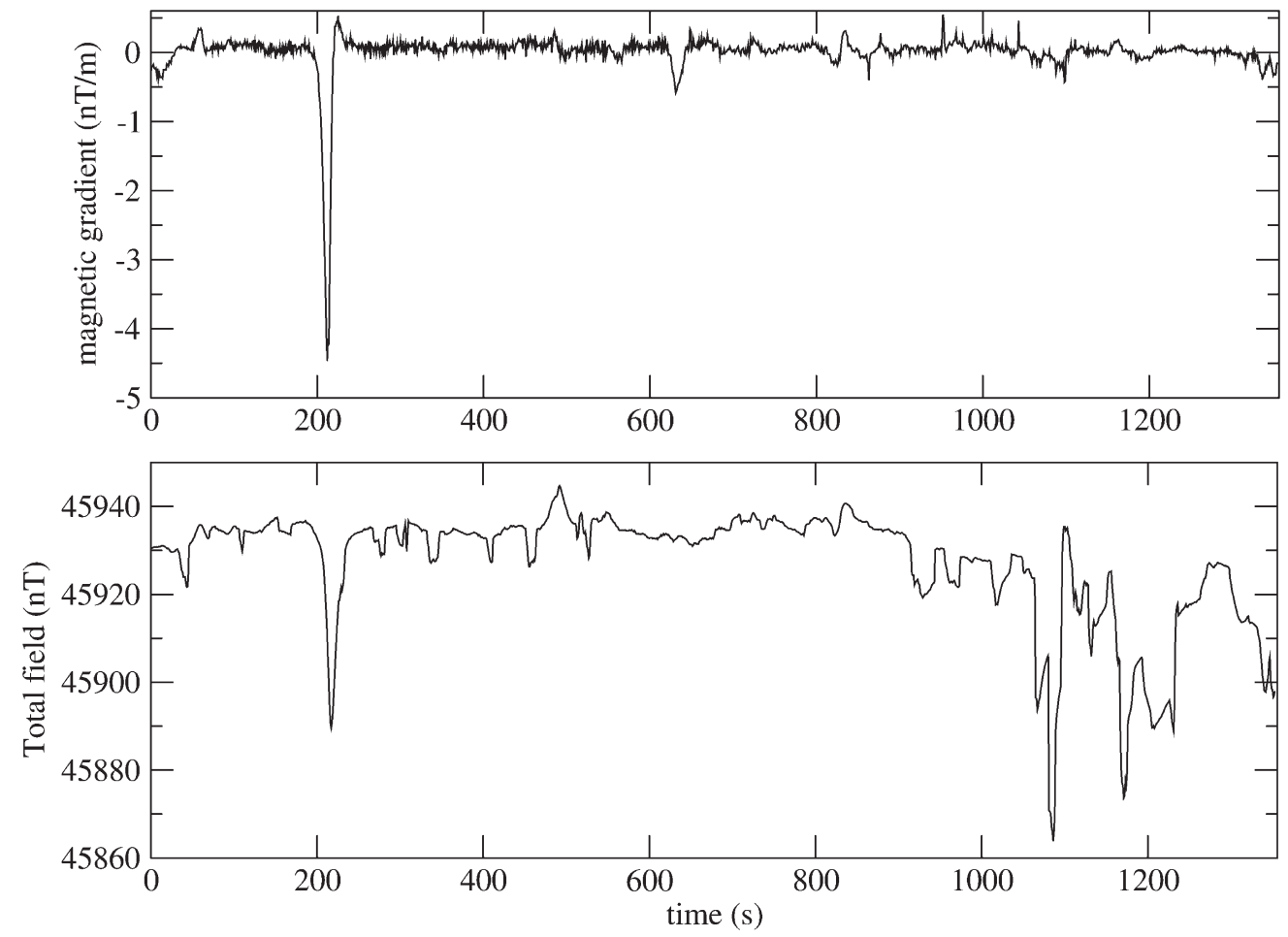

Fig. 5. Example of the magnetic signal (bottom panel) and gradient signal (upper panel) along a survey line. The large anomaly on the left is due to some magnetic sources, while the anomalies on the right, which are present only on the magnetic signal and not in the gradient, are probably due to anthropic noise.

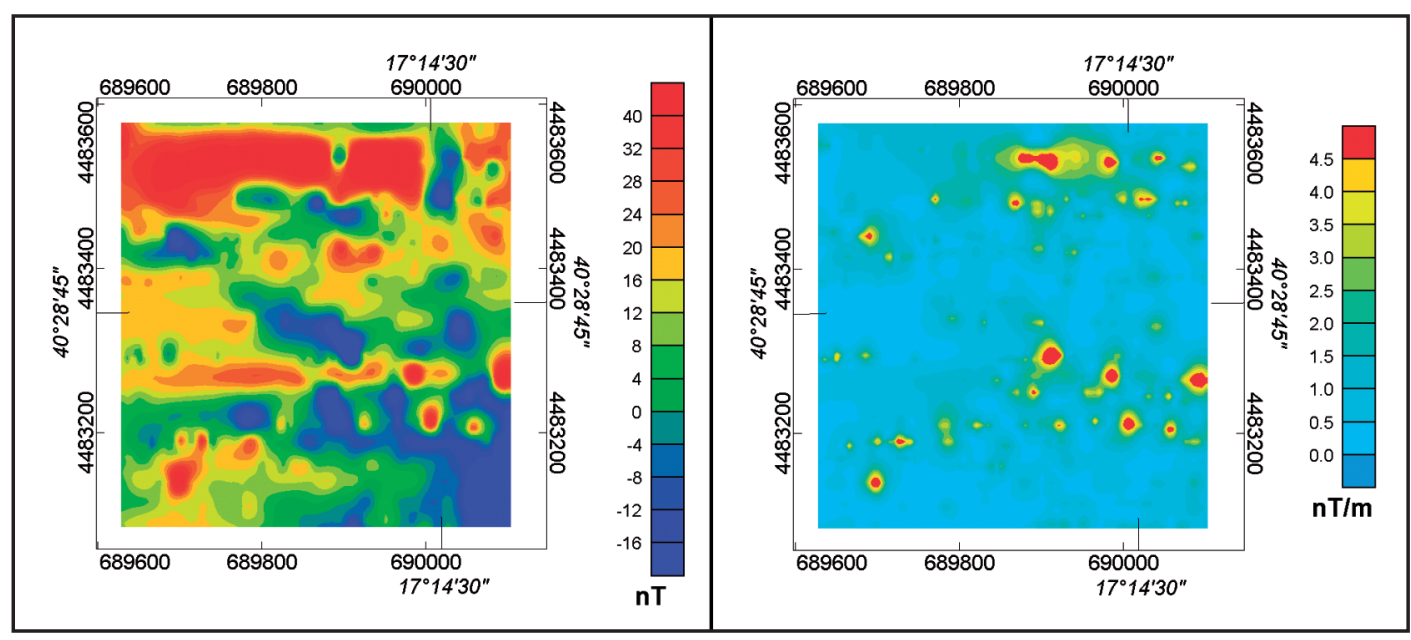

Fig. 6. Left panel: magnetic anomaly map in the restricted boxed area. Right panel: example of a 3D magnetic analytic signal evaluated by computing the numerical derivative of the magnetic data. 

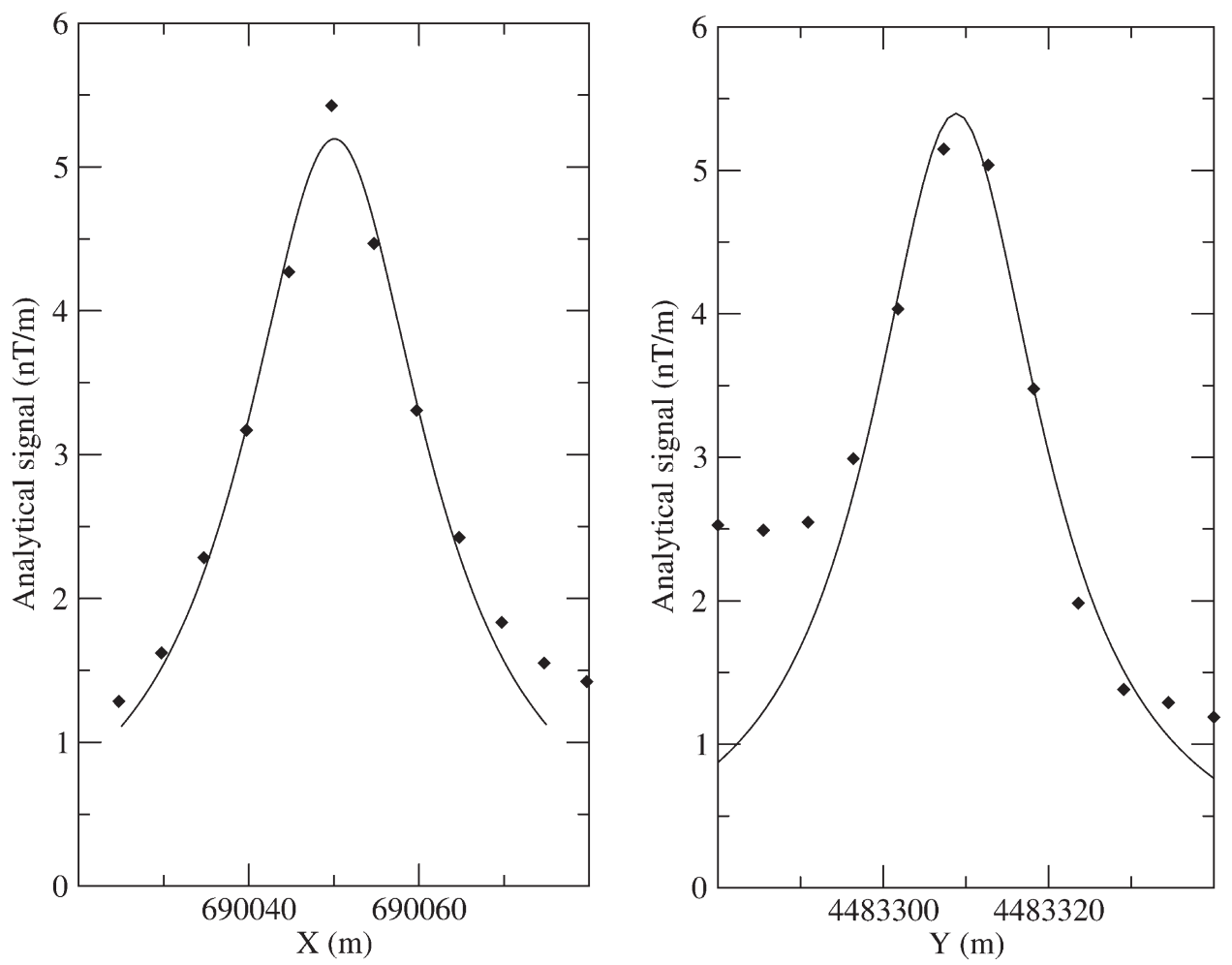

Fig. 7. Analytical signal least squares fit with a bell shaped function in order to estimate the depth of the source of anomaly n. 3 .

Again, we used the measured $M_{x}$ and $M_{y}$ data to construct the vertical component on a square grid using a relation (eq. 3.2) that is a mathematically stable operator (Nabighian, 1984).

Study of the analytic signal properties in a gradiometric survey can reveal additional information on the magnetised sources. In the case of a sharp and localised metallic target, the analytic signal profile has a bell shape, where the half width at half height is equal to the depth of the anomalous magnetic contrast. An example of this shape is plotted in fig. 7 for anomaly n. 3; the least squares fit with a bell shaped function indicates a depth of ca. $13 \mathrm{~m}$, which is compatible with the object on the seabed found by visual investigation (table I). This technique can thus be useful in searching for objects hidden from a visual inspection because they are buried in the seabed mud; the accurate processing of analytical signal data can help to reveal the depth at which to search for these buried objects.

\section{Conclusions}

We demonstrate how a gradiometric configuration can be useful to resolve magnetic anomalies in a noisy anthropic area. We were able to clearly identify a large number of magnetic targets deployed on the seabed. Although the results of the present survey are positive as a whole, need for a dedicated structure is highlighted: a specific setup that can guarantee better stability for the towing system for optimal use in similar contexts must be developed. In fact, the towed rubber dinghy induced a tilt 
movement in the sensor device that generated the induction of additional high frequency noise. As a result of this experience, and for use of the device for both environmental and archaeological surveys, a suitable catamaran structure has been designed and developed, which gives more satisfactory results. Furthermore, the total magnetic field is by definition a scalar physical quantity, and its space derivative is a three-dimensional vector; the gradiometer configuration used is only able to measure the projection of the gradient along the direction of separation of the two sensors. To measure all of the vectorial components and therefore the whole analytical signal at the same time, it would be necessary to acquire three independent axial measures using four sensors placed on an dedicated platform; this would ensure that the whole analytic signal could be measured simultaneously with significant survey time savings. In this work, we overcame this problem using only a couple of sensors by doing lines and ties, thus doubling the time necessary for the survey while still maintaining a compatible confidence level on data. The results here obtained clearly demonstrate that even when measuring only two components of the magnetic gradient we were able to provide good information for the locating metallic targets in a noisy area such as the Taranto harbour.

\section{REFERENCES}

Boyce, J. and E.G. Reinhardt (2001): Marine magnetic survey of a submerged Roman Harbour, Caesarea Maritima, Israel, International Journal of Nautical Archaeology, 33 (1), 122-136

Caratori Tontini, F., C. Carmisciano, M. Ciminale, M. Grassi, P. Lusiani, S. Monti and P. Stefanelli (2006): High resolution marine magnetic surveys for searching underwater cultural resources, Annals of Geophysics, 49 (6), 1177-1185.

Hrvorc, D. and M.R. PozZA (2006): Mapping marine ferrous targets using real-time $3 \mathrm{D}$ analytical signal data, The leading Edge, 25, 54-56.

Mansoor, N., L. Slater, F. Artigas and E. Auken (2006): High-resolution geophysical characterization of shallow water wetlands, Geophysics, 71, 4, B101-B109.

Ministero AMBIENTE E TUTELA TERRITORIO (2001): Decreto ministeriale n. 468, annex B, 18 September 2001, «Programma nazionale di bonifica e ripristino ambientale dei siti inquinati», Gazz. Uff., January 16, 2002, n. 13 supp. ord.

NABIGHIAN, M.N. (1972): The analytical signal of two-dimensional magnetic bodies with polygonal cross section: its properties and use for automated anomaly interpretation, Geophysics, 37, 507-517.

NABIGHIAN, M.N. (1974): Additional comment on the analytical signal of two dimensional magnetic bodies with polygonal cross section, Geophysics, 39, 85-92.

NABIGHIAN, M.N. (1984): Toward a three-dimensional automatic interpretation of potential field data via generalized Hilbert transforms: Fundamental relations, Geophysics, 49, 780-786.

NELSON, J.B. (1986): An alternate derivation of the three dimensional Hilbert transform relations from first principles, Geophysics, 51 , 1014-1015.

Parlamento italiano (1998): Art. 1, comma 4, Legge December 9,1998 , n. 426 «Nuovi interventi in campo ambientale», Gazz. Uff., December 14, 1998, n. 291

Pedersen, L.B. (1989): Relations between horizontal and vertical gradients of potential fields, Geophysics, $\mathbf{5 4}$ (5), 662-663,

PozZA, M.R., J.I. BoyCE and W.A. Morris (2004): Lakebased magnetic mapping of contaminated sediment distribution, Hamilton Harbour, Lake Ontario, Canada, Journal of Applied Geophysics, 57, 23-41.

RAVAT, D. (1996): Magnetic properties of unrusted steel drums from laboratory and field-magnetic measurements, Geophysics, 61 (5), 1325-1335.

Roest, W.R., J Verhoef and M. Pilkington (1992): Magnetic interpretation using the 3D analytic signal, Geophysics, 57, 116-125.

Weiss, E., B. Ginzburg, T.R. Coehn, H. ZafriR, R. Alimi, N. SALOMONSKI and J. SHARVIT (2007): High resolution Marine magnetic survey of shallow water littoral area, Sensors, 7, 1679-1712.

(received, May 13, 2008; accepted, June 15, 2009) 